\title{
SIMULATION AND OPTIMIZATION OF A CHP BIOMASS PLANT AND DISTRICT HEATING NETWORK
}

\author{
K. Sartor ${ }^{\mathrm{a}, *}$, S. Quoilin ${ }^{\mathrm{a}}$, P. Dewallef ${ }^{\mathrm{a}}$ \\ ${ }^{a}$ Department of Aerospace and Mechanics, Thermodynamics Laboratory, University of Liège, Chemin des Chevreuils 7 , \\ B-4000 Liège, Belgium
}

\begin{abstract}
Biomass Combined Heat and Power (CHP) plants connected to district heating (DH) networks are recognized nowadays as a very good opportunity to increase the share of renewable sources into energy systems. However, as CHP plants are not optimized for electricity production, their operation is profitable only if a sufficient heat demand is available throughout the year. Most of the time, pre-feasibility studies are based on peak power demand and business plans only assume monthly or yearly consumption data. This approach usually turns out to overestimate the number of operating hours or oversize the plant capacity.

This contribution presents a methodology intended to be simple and effective that provides accurate estimations of economical, environmental and energetic performances of CHP plants connected to district heating networks. A quasi-steady state simulation model of a CHP plant combined with a simulation model of the district heating network installed on the Campus of the University in Liège (Belgium) is used as an application framework to demonstrate the effectiveness of the selected approach. Based on the developed model and actual consumption data, several scenarios for energy savings are considered and ranked.
\end{abstract}

The potential energy savings and resulting energy costs are estimated enabling more general conclusions to be drawn on the opportunity of using district heating networks in urban districts for Western Europe countries.

Keywords: Biomass, Combined Heat and Power, Power Plant, DHN, District Heating Network, Wood Pellet.

\footnotetext{
*Corresponding author: kevin.sartor@ulg.ac.be; +32(0)4 366.48.20

Email addresses: kevin. sartor@ulg.ac.be (K. Sartor), squoilin@ulg.ac.be (S. Quoilin), p. dewallef@ulg.ac.be (P. Dewallef)

Preprint submitted to Elsevier
} 


\title{
Nomenclature
}

\author{
Acronyms \\ CHP Combined Heat and Power \\ $\mathrm{COH}$ Cost of Heat ( $€$ per MWh) \\ DH District Heating \\ LHV Low Heating Value \\ $N G \quad$ Natural gas \\ $U L g \quad$ University of Liège
}

\section{Roman symbols}

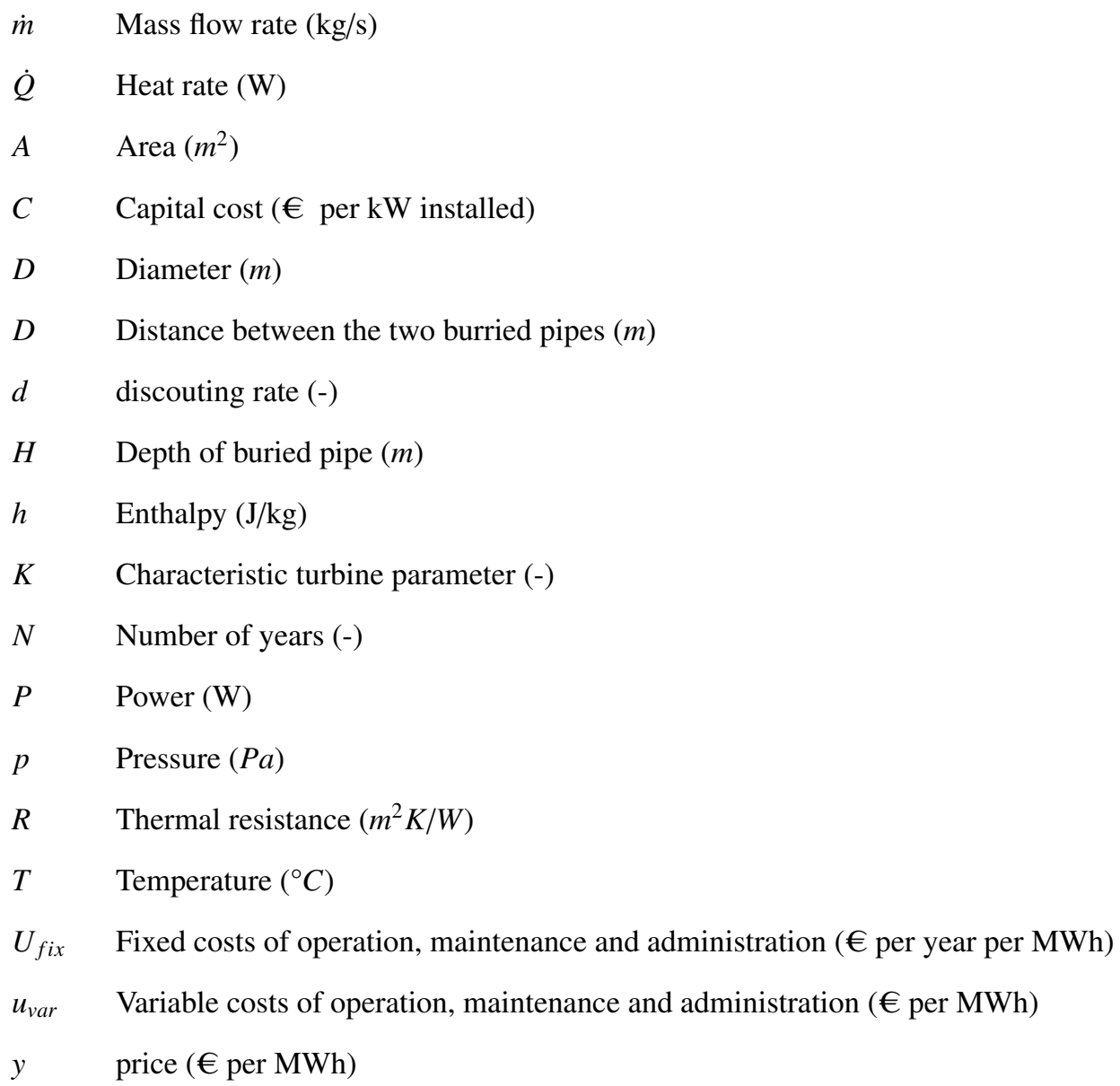

\section{Greek symbols}

$\Delta T_{l m} \quad$ Logarithmic mean temperature difference $\left({ }^{\circ} \mathrm{C}\right)$ 


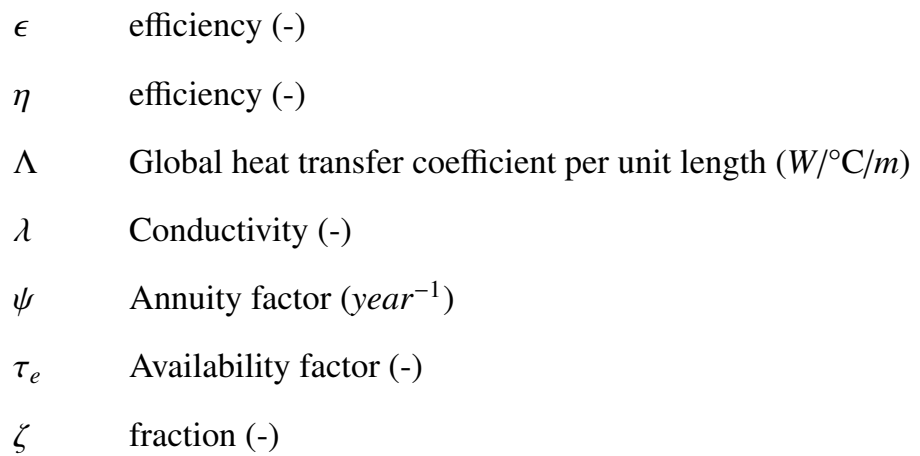

\section{Subscripts}

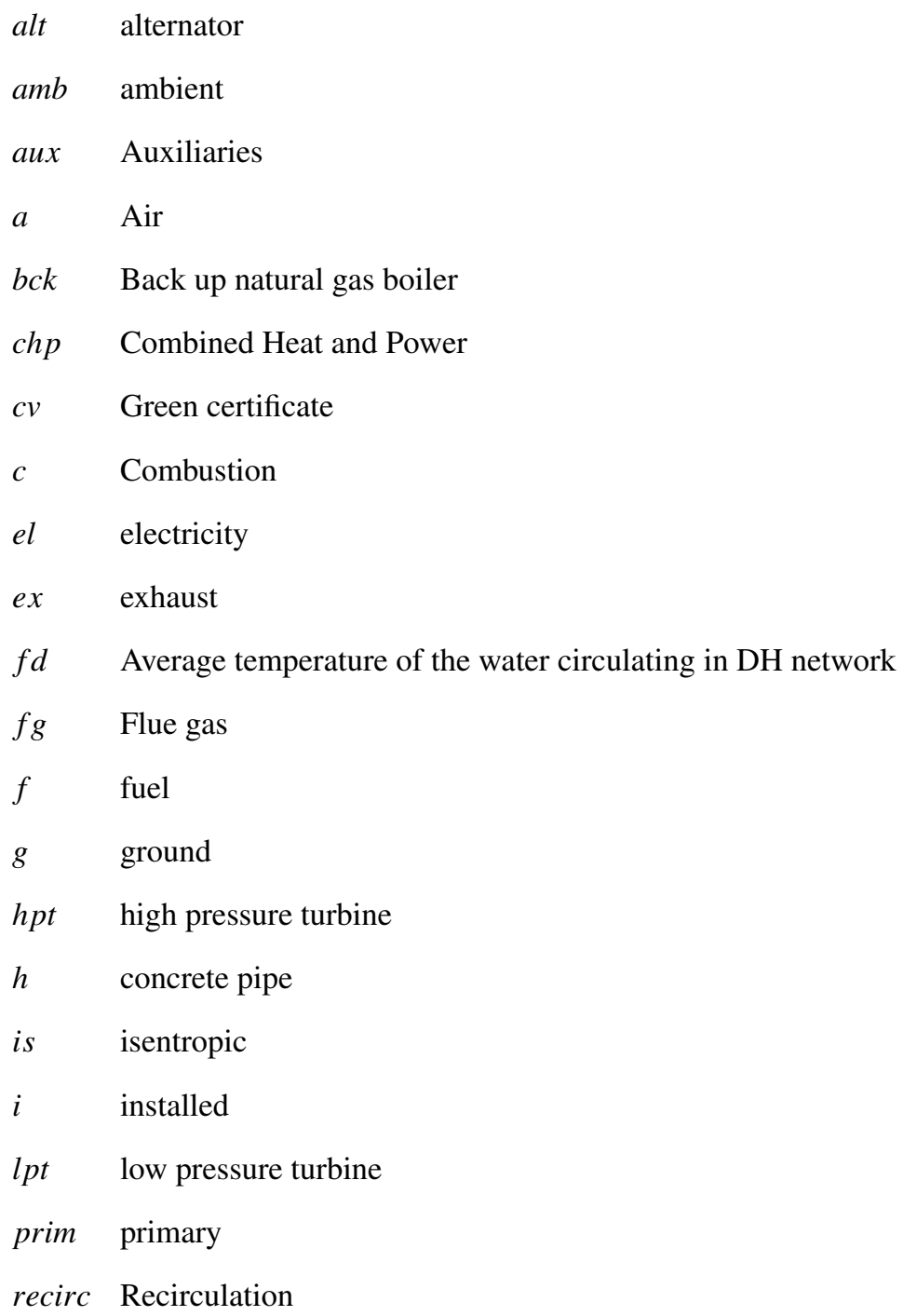




$\begin{array}{ll}r r & \text { Surface of return pipe } \\ r & \text { Return pipe } \\ r & \text { return pipe } \\ s s & \text { Surface of supply pipe } \\ s t & \text { steam } \\ s u & \text { supply } \\ s & \text { supply pipe } \\ t h & \text { thermal } \\ w & \text { wall }\end{array}$

\section{Introduction}

Biomass CHP plants are often seen as an efficient way to reduce greenhouse gases emissions due to their very low $\mathrm{CO}_{2}$ emissions level $[1,2]$. Biomass pellet price per unit of primary energy is also lower than that of fossil fuels (e.g. 36.55 against $55.9 €$ per MWh [3] for natural gas). Another attractive aspect of biomass lies in the fact that the resource is more equally spread over the earth which can be an advantage from the energy independence point of view. However, due to the nature and the diversity of the biomass fuels, achieving high efficiency together with clean combustion is far more difficult for biomass than for traditional fossil fuels. Therefore, the investment required to build a biomass CHP plant is significantly higher than that of a corresponding natural gas CHP plant based on reciprocating engines or gas turbines.

Because of the significant cost, the conversion efficiency of the primary energy into electricity and heat must be maintained to a maximum during the maximum number of hours throughout the year. Practically, this means that the heat produced by the CHP plant must be useful to some consumers for some minimum number of hours per year. The assessment of the threshold below which the cost of heat from the CHP is above the one generated by a traditional natural gas boiler depends upon the application at hand and is addressed in the following. Clearly, industries needing a steady and important amount of heat throughout the year are the best case for biomass CHP plants, yet most of these industries are already equipped with CHP plants of some kind. A developing idea is to use the heat produced by a CHP plant in district heating networks. The practice of district heating networks is not new and installations were realized throughout Europe for several decades (mostly Germany, Scandinavian countries and Eastern Europe countries).

The new context of rising price of fossil fuels and pressure towards lower greenhouse gases emissions shed a new light on district heating networks and lots of new such installations have been realized during the last 10 years across the whole Europe. The attractive property of a CHP plant connected to a district heating network is the possibility to massively include renewable sources of energy into energy systems at a reasonable cost.

However, one must always keep in mind that even if a DH network enables the heat to be transported over long distances (typically several kilometers) which therefore guarantees a large amount of heat demand, the load profile throughout the year is far from being constant. In these 
conditions, a good practice consists in designing the CHP plant on the minimum heat demand. However, in the case of DH networks, the minimum heat demand is often very low and does not justify the installation of a CHP plant. Finding the right size and technology for CHP plant connected to DH networks that minimizes the cost of heat turns out to be a delicate exercise requiring a careful estimation of both the average conversion efficiencies for electricity/heat production and the equivalent utilization time of the plant at rated output ${ }^{1}$.

The high level of conversion efficiencies (in the range 70-90\% [4]) combined to the allocation of subsidies enables a significant decrease of the heat cost. However, at the level of feasibility studies, rule of thumbs based on existing installations are often used which does not guarantee a competitive cost of heat. While methodologies exist to integrate CHP systems, the considered conversion efficiencies are most of the time based on correlation from existing plants and whose generalization is not guaranteed (e.g., [5, 6]). Some more detailed models, even dynamic models, can be found in the literature [7], yet their integration on the basis of the demand curve along a typical year is not considered.

The scope of this contribution is to present a simple, yet complete, methodology to simulate a biomass CHP plant based on a steam cycle with extraction turbines and connected to a DH network. The simulation model is used to assess the average conversion efficiencies over a complete year of operation, thus providing reliable estimates for energy cost predictions. The methodology is applied to an existing installation available on the University campus in Liege (Belgium) for which consumption data is available. The example application enables the estimation of meaningful cost and environmental parameters related to the use of such an installation.

\section{Problem statement}

When using a CHP plant to feed a DH network, the goal is to optimize the conversion efficiency of the primary energy into useful energy (electricity and heat). If $P_{e l, c h p}$ and $P_{t h, c h p}$ represent respectively the electrical and thermal power generated by the CHP plant and $P_{\text {prim,chp }}$ denotes the primary energy used to generate $P_{e l, c h p}$ and $P_{t h, c h p}$, the electrical and thermal efficiencies are defined respectively as:

$$
\eta_{e l, c h p}=\frac{P_{e l, c h p}}{P_{\text {prim }, \text { chp }}} \text { and } \eta_{t h, c h p}=\frac{P_{t h, c h p}}{P_{\text {prim }, \text { ch } p}}
$$

The total CHP efficiency is defined as $\eta_{c h p}=\eta_{e l, c h p}+\eta_{t h, c h p}$. Burning one kilowatthour $(\mathrm{kWh})$ of natural gas releases $251 \mathrm{~g}$ of $\mathrm{CO}_{2}{ }^{2}$ while the same primary energy of biomass releases between 0 and $30 \mathrm{~g}$ of $\mathrm{CO}_{2}$ only. Biomass fuels can be of very different types and it is beyond the scope of the present contribution to list them all and it will be assumed that the biomass fuel used herein generates $30 \mathrm{~g}$ of $\mathrm{CO}_{2}$ per kilowatt-hour of primary energy. Most of the European countries have policies to reward the use of biomass fuels under the form of subsidies or premium on the energy produced from biomass fuels. More recently, other methodologies such as life cycle analysis take into account the whole production process from the construction of the plant to its decommissioning to assess the actual saving in terms of $\mathrm{CO}_{2}$ emissions.

\footnotetext{
${ }^{1}$ The equivalent utilization time at rated output is the energy generated by the plant in a period of time divided by the electrical energy which could be produced by the plant running the whole year at rated output.

${ }^{2}$ By convention the primary energy is based on the higher heating value rather on the lower heating value.
} 
However, the most critical and decisive criteria for the development of such technologies is the cost of the energy. The problem of optimizing a CHP plant connected to a DH network is considered herein as designing the CHP plant minimizing cost of heat supplied to the user. To that end, a cost model for the heat production must be designed which takes into account the cost of capital, the fuel costs, the operation and maintenance cost as well as the selling price of the electricity produced. The previous consideration translates the fact that the plant is supposed to be heat-driven, the electricity being considered as a by-product sold to the grid operator or consumed locally.

The cost model per unit of thermal energy used herein is derived similarly to the one defined for electricity in [8]. According to this model, the cost of heat is expressed as:

$$
C O H=\underbrace{\frac{C \cdot \psi+U_{f i x}}{P_{i, t h, c h p} \cdot \tau_{e}}}_{\text {Fixed cost }}+\underbrace{\frac{y_{f}}{\bar{\eta}_{t h, c h p}}+u_{v a r}}_{\text {Variable cost }}-\underbrace{\left(y_{e l}+\tau_{c v} y_{c v}\right) \frac{\bar{\eta}_{e l, c h p}}{\bar{\eta}_{t h, c h p}}}_{\text {Elec. selling price }}
$$

where $C$ is the total investment cost, $\psi$ is the annuity factor which takes into account the present value of money and represents the annual repayment for the initial investment expressed in year $^{-1}$. The annuity factor is assessed according to:

$$
\psi=\frac{d}{1-(1+d)^{-N}}
$$

where $d$ is the discounting rate per year and $N$ the number of years for which the installation is used (e.g., the life time of the plant). $P_{i, t h, c h p}$ is the installed thermal power of the CHP plant in MW and $\tau_{e}$ is the equivalent utilization time at rated power output. $\tau_{e}$ embeds the availability factor of the plant (around $92 \%$ for a biomass CHP plant). $y_{f}$ is the cost of fuel in $€ / \mathrm{MWh}$, $U_{f i x}$ is the fixed cost of operation, maintenance and administration in $€ /$ year and $u_{v a r}$ is the variable cost of operation, maintenance and repair in $€ / \mathrm{MWh} . \bar{\eta}_{t h, c h p}$ is the annual average thermal efficiency defined similarly to $\eta_{t h, c h p}$ yet taking into account the start/stop procedures (if any) and the part load efficiency. $y_{e l}$ is the price of electricity in $€ / \mathrm{MWh}$ while $\tau_{c v}$ and $y_{c v}$ are respectively the number of green certificates per MWh of electricity produced ${ }^{3}$ and the unit price of green certificate. The term $\tau_{c v} y_{c v}$ is replaced by the premium on the electricity selling when feed-in tariffs are used instead.

The determination of $C, U_{f i x}, u_{v a r}, d$ and $N$ is not within the scope of the present contribution and reliable estimates can be found e.g., in $[9,8]$. Neither the influence of fuel cost $y_{f}$ nor the one of the supporting policies $\tau_{c v} y_{c v}$ will be long discussed herein and representative value of the market in Belgium will be used, as it is relatively straightforward for the reader to include his proper data into the above model.

However, the determination of $\tau_{e}, \bar{\eta}_{e, c h p}$ and $\bar{\eta}_{t h, c h p}$ is not straightforward and very often overlooked as these values strongly depend upon the size of the CHP plant and the time profile of heat demand. Studying how the CHP plant matches the heat demand and assessing the resulting performance is the main scope of the present contribution. In order to support the discussion, it

\footnotetext{
${ }^{3}$ For the Walloon region of Belgium one green certificate is granted for every $456 \mathrm{~kg}$ of $\mathrm{CO}_{2}$ saving. A maximum of 2 green certificate is allowed per MWh of electricity produced.
} 
is insightful to study the influence of $\tau_{e}$ on the cost of heat for two typical situations, namely a $\mathrm{CHP}$ plant and a natural gas boiler. The two $\mathrm{COH}$ are presented in Figure 1 for a wide range of $\tau_{e}$. It can readily be seen that for a natural gas boiler the cost of heat is nearly constant since fixed costs are negligible compared to variable costs. For the CHP plant however, the fixed costs are far more important and create a strong dependency between the cost of heat and the utilization time.

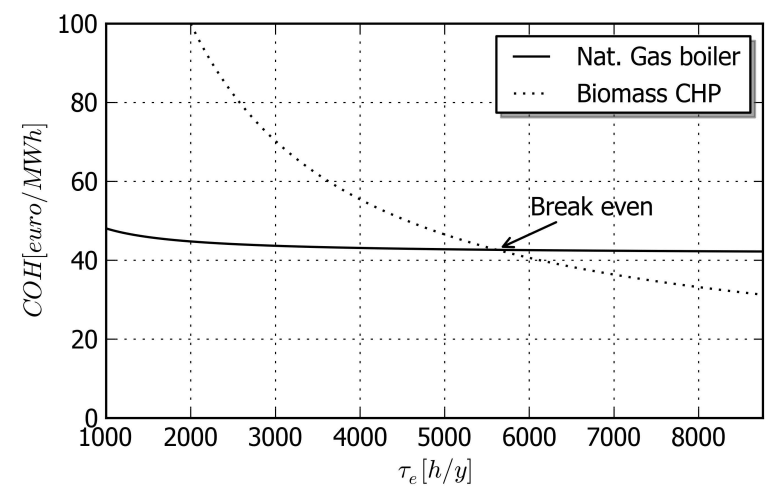

Figure 1: Influence of the equivalent utilization time on the cost of heat.

When connected to a DH network, the CHP plant is designed to supply the base load while backup boilers supply the peak load. The situation is depicted in Figure 2 representing the evolution of a building heating load during the day. The area denoted $E_{t h, c h p}$ (light grey) represents the heat supplied by the CHP below the installed thermal power while the area $E_{t h, b c k}$ (dark grey) is for the thermal energy supplied by the backup boilers.

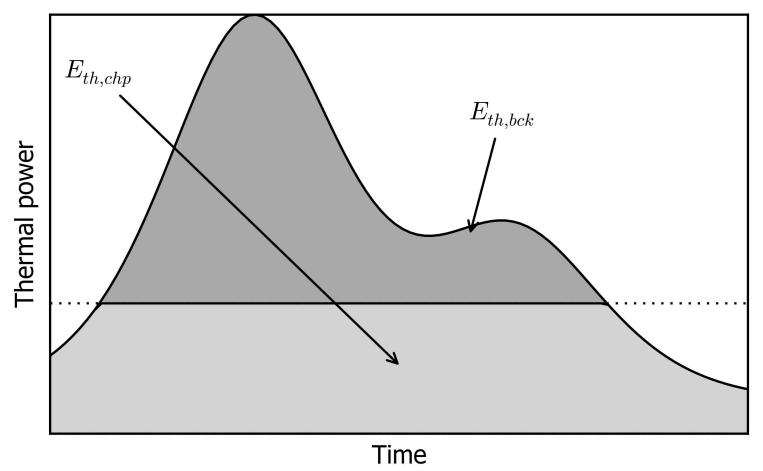

Figure 2: Variation of load during the day for a typical heating application

When integrating the scheme on the whole year, the total heat demand can be represented as a cumulative load diagram such as the one represented in Figure 3.

Such a diagram is very useful to assess the equivalent utilization time corresponding to a specific installed thermal power as well as the thermal energy provided by a CHP plant given a specific thermal demand. In Figure 3, the thermal energy supplied by the CHP plant is the light grey area. The remaining area under the demand curve (dark grey) is the amount of heat supplied by the backup boiler. The equivalent utilization time $\tau_{e}$ used in equations (2) is obtained by dividing the light grey area (expressed in MWh) by the installed power (expressed in MW) 


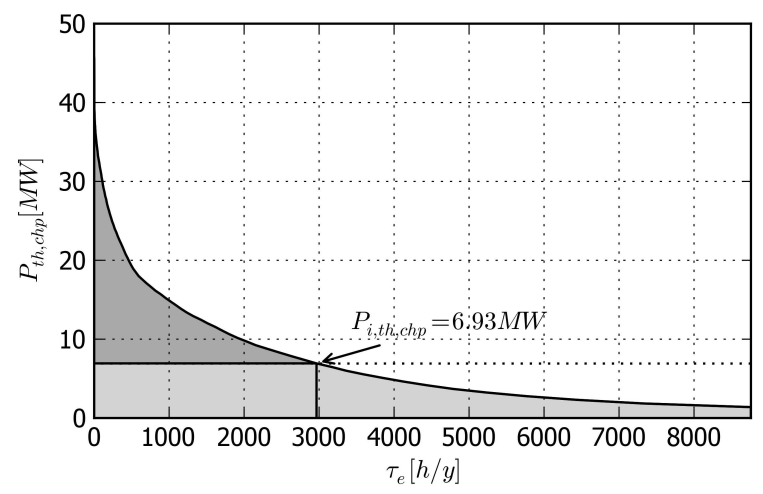

Figure 3: Cumulative demand curve of a district heating network

and multiplying the result by the reliability ratio of the plant (typically $98 \%$ ). The time step used for the simulation presented herein is one hour. It is however too coarse to catch the detailed behaviour of the plant and a further refinement will consist in reducing the time step to 15 minutes or less if transient effects are taken into account.

If the costs of heat generated through relation (2) for the CHP plant and backup boiler are denoted respectively by $\mathrm{COH}_{c h p}$ and $\mathrm{COH}_{b c k}$ and $\Theta$ is the ratio of the thermal energy generated by the CHP plant to the total thermal energy for the considered time interval, the average cost of heat is assessed through:

$$
C O H=\Theta C O H_{c h p}+(1-\Theta) C O H_{b c k}
$$

In addition to the cost model summarized by relation (2), it is interesting to study the influence of the heat losses due to the DH network and to express the cost of heat per unit of heat delivered to the customer. This is achieved through the definition of a transport efficiency $\xi_{D H N}$ defined as the ratio of the heat delivered to the consumer to the heat produced by the plant. With this tranport efficiency, relation (2) yields:

$$
\begin{array}{r}
C O H=\frac{1}{\xi_{D H N}}\left[\frac{C \cdot \psi+U_{f i x}}{P_{i, t h, c h p} \cdot \tau_{e}}+\frac{y_{f}}{\bar{\eta}_{t h, c h p}}+u_{v a r}\right. \\
\left.-\left(y_{e l}+\tau_{c v} y_{c v}\right) \frac{\bar{\eta}_{e l, c h p}}{\bar{\eta}_{t h, c h p}}\right]
\end{array}
$$

\section{The simulation model}

Section 2 aimed at assessing the exact amount of heat supplied by a CHP plant to a DH network whose heat demand profile is known. The scope of the present section is to introduce a more detailed simulation model to assess the thermal and electricity conversion efficiencies during the whole year, thus taking into account off-design ambient conditions and part load operation of the plant. 
The considered biomass CHP plant consists of a biomass furnace connected to a boiler made of an economizer, an evaporator and super-heater section. A schematic of the cycle is represented in Figure 4.

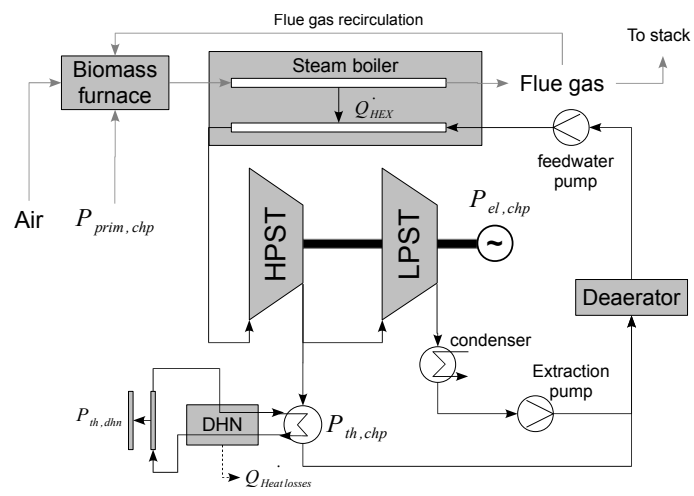

Figure 4: Schematic of CHP plant

In essence, the complete simulation model of the plant is made of basic 0-dimensional components where the relation input-output satisfies the energy, mass and momentum balances. The components mentioned in Figure 4 are detailed in the next subsections.

\subsection{Biomass combustion}

The model developed is intended to predict the thermodynamic state of the flue gas at the exhaust of the furnace. Assuming that both combustion air and flue gases can be modelled by perfect gas and neglecting pressure losses, the temperature of the flue gas can be assessed by the resolution of the mass and energy balance. Basically, the approach considered herein is the one advised in [10] to which several modifications have been made to take into account combustion air humidity and flue gas recirculation.

When introducing flue gas recirculation, the mass and energy balance equations yield:

$$
\begin{aligned}
\dot{m}_{f g}= & \dot{m}_{f g, \text { recirc }}+\dot{m}_{a}+\dot{m}_{f} \\
\dot{m}_{f g} h_{f g}= & \dot{m}_{f g, \text { recirc }} h_{f g}+\dot{m}_{a} \cdot h_{a} \\
& +\dot{m}_{f}\left(h_{f}+\varepsilon_{c} L H V_{f}\right)
\end{aligned}
$$

where the suffix $a, f$ and $f g$ refer respectively to combustion air, fuel and flue gases. $\dot{m}$ stands for the mass flow rate, $h$ is the enthalpy, $L H V_{f}$ is the fuel lower heating value and $\varepsilon_{c}$ is the combustion efficiency. The resolution of the above equations requires thermodynamic properties of the flue gases. For fossil fuels, such properties are available for several values of the excess air but when dealing with biomass whose composition is highly variable, a combustion model is needed to assess the thermodynamic properties of the flue gas.

Again, the combustion model is inspired from the one advised in [10] where complete combustion of the biomass is assumed. This is compatible with the high air excess commonly used in biomass boilers where $C O$ emissions are most of the time very low. This model also neglects the $\mathrm{SO}_{3}$ formation and assumes that sulphur is completely transformed into $\mathrm{SO}_{2}$. The biomass composition is handled through a general model $C_{m} H_{n} O_{x} N_{y} S_{z}$ where the subscripts are the ratio between wet basis mass fraction of each component to its molar mass. This proposed biomass composition is easily determined if biomass is normalized (EN 15104, EN 14774, EN 14775, 
EN 15289). Others components like $\mathrm{Cl}, \mathrm{Cr}, \ldots$ are neglected while EN 14961-2 involves their content inside sold biomass is less than $1 \%$.

The formulation proposed in [10] is extended to take into account air humidity (Eq 9).

$$
\begin{aligned}
& \underbrace{C_{m} H_{n} O_{x} N_{y} S_{z}+x_{w} \cdot H_{2} O}_{\text {A mole of wet biomass }}+(1+e) \cdot \kappa \cdot\left(O_{2}+\beta \cdot N_{2}+\gamma \cdot H_{2} O\right) \Rightarrow \\
& m \cdot C O_{2}+\left(\frac{n}{2}+x_{w}+\gamma\right) \cdot H_{2} O+\left(\beta \cdot \kappa+\frac{y}{2}\right) \cdot N_{2}+e \cdot \kappa \cdot O_{2}+z \cdot S O_{2}
\end{aligned}
$$

In the above equation $e$ is the excess air, $x_{w}$ is the mole fraction of water in biomass, $\beta$ is the ratio between molar fraction of nitrogen and oxygen in wet air, $\gamma$ is the molar fraction between water and oxygen in wet air and $\kappa=\left(m+\frac{n}{4}-\frac{x}{2}+z\right)$. The ashes are considered as neutral for combustion reaction. As flue gas recirculation will be investigated further, the above combustion equation can be extended to take into account the flue gas recirculation which can be done by adding the following term to both members of the combustion equation (9):

$$
\chi \cdot\left[m \cdot \mathrm{CO}_{2}+\left(\frac{n}{2}+x_{w}+\gamma\right) \cdot \mathrm{H}_{2} \mathrm{O}+\left(\beta \cdot \kappa+\frac{y}{2}\right) \cdot N_{2}+e \cdot \kappa \cdot O_{2}+z \cdot S O_{2}\right]
$$

where $\chi$ is defined as the molar fraction of recirculated combustion gases. In this case, ashes are not recirculate as they are captured before recirculation.

For given ambient conditions, excess air, biomass composition and mass flow rate and the above equations allow the determination of flues gas thermodynamic properties for any given furnace exhaust temperature. The advantage of the developed model, is the high flexibility regarding biomass fuel and operating conditions allowing accurate prediction of the boiler efficiency.

\subsection{Steam boiler}

Once the thermodynamic properties of the flue gas at the exhaust of the furnace are known, the heat transferred from the flue gases to produce the steam must be determined. The energy balance together with the calculation of the heat transfer between the water and the flue gases can be used which gives the following balance equations:

$$
\begin{aligned}
& \dot{Q}_{H E X}=\dot{m}_{f g}\left(h_{s u, f g}-h_{e x, f g}\right) \\
& \dot{Q}_{H E X}=\dot{m}_{s t}\left(h_{e x, s t}-h_{s u, s t}\right) \\
& \dot{Q}_{H E X}=\int_{A} U_{l o c}\left(T_{f g}-T_{s t}\right) d A=A U \Delta T_{l m}
\end{aligned}
$$

where $\dot{Q}_{H E X}$ is the rate of heat transferred from the flue gas to the steam, $\dot{m}_{f g}$ the flue gas mass flow rate determined by the furnace model, $\dot{m}_{s t}$ is the steam mass flow rate, $h_{s u, f g}$ and $h_{s u, s t}$ are the known enthalpy of the flue gas and steam at the boiler supply. $A$ is the exchange area of the heat exchanger, $U$ the average overall heat transfer coefficient and $\Delta T_{l m}$ is the logarithmic mean temperature difference between the water and the flue gas (see [11] for further details and definitions). As water undergoes a phase change, the heat transfer coefficient $U$ cannot be considered constant for the whole boiler and the heat exchanger is split up into three parts, 
namely the economizer for the heating of subcooled water, the evaporator for the wet steam and the superheater for the superheated steam.

For sake of robustness, the number of transfer unit (NTU) has been used instead of the logarithmic mean temperature difference. When the product $A U$ is known and given a set point on the steam mass flow rate, the exhaust temperatures for the flue gas and steam can be calculated thus enabling the assessment of the boiler efficiency. This is an interesting feature of this simple model as boiler efficiency can be assessed when the boiler operates outside nominal conditions.

Practically, $A U$ can be determined from the heat exchanger geometry and existing correlations (see [12]) or be determined from the value of the pinch point for nominal operation. In the application presented herein, the latter approach has been selected since it was the only data available.

Additionally to the rate of heat exchanged, the boiler model is completed by a prediction of the heat exchangers pressure drop which enables the calculation of the fan consumption. If experimental data are not available, some empirical correlations could be used to determine these pressure drops [13]. It has to be noted that the heat exchanger transferring the heat from the steam to the DH network is also modelled by the same methodology.

\subsection{Steam turbines}

In the above section, the mass flow rate was assumed to be known. In effect, the effective mass flow rate is obtained by adapting the feedwater pressure to the turbine permeability. Indeed, the steam turbine can be seen as a nozzle whose mass flow rate depends upon the inlet pressure and temperature and outlet pressure. The dependency between these parameters is expressed by the so-called Stodola line [14] expressed below:

$$
\frac{\dot{m}_{s t} \sqrt{T_{s u, s t}}}{\sqrt{p_{s u, s t}^{2}-p_{e x, s t}^{2}}}=K
$$

The parameter $K$ is constant for a very wide range of operation and therefore can be determined from the nominal operation. Once, $K$ is determined, the mass flow rate can be calculated from the inlet pressure and temperature together with the pressure drop across the turbine. The gross electrical power delivered by the turbine is given by:

$$
P_{e l, t u r b}=\dot{m}_{s t} \cdot \eta_{a l t} \cdot \varepsilon_{i s} \cdot\left(h_{s u, s t}-h_{e x, s, s t}\right)
$$

where $\eta_{\text {alt }}$ is the alternator efficiency, $\varepsilon_{i s}$ is the isentropic efficiency of the turbine and $h_{e x, s, s t}$ the enthalpy at the exhaust of the turbine resulting from an isentropic expansion from $p_{s u}$ to $p_{e x}$. The isentropic efficiency is considered constant on the whole operating range of the turbine. The latter assumption does not strictly holds and the efficiency decreases with the mass flow rate. However, the decrease in efficiency is limited in the operating range of the high pressure turbine and is only noticeable for the low pressure turbine when an important steam flow is extracted for the disctrict heating network.

The net electrical power generated is assessed by:

$$
P_{e l, c h p}=\dot{m}_{s t} \eta_{a l t}\left[\varepsilon_{i s, h p t} \cdot\left(h_{s u, h p t}-h_{e x, s, h p t}\right)+(1-\zeta) \varepsilon_{i s, l p t} \cdot\left(h_{s u, l p t}-h_{e x, s, l p t}\right)\right]-P_{a u x}
$$




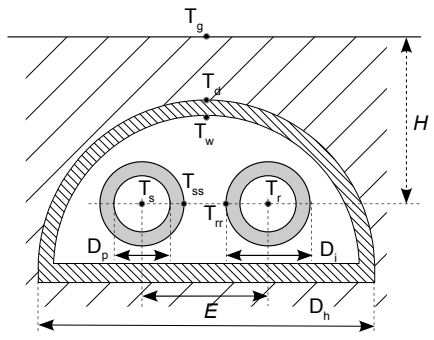

(a) Cross-section diagram

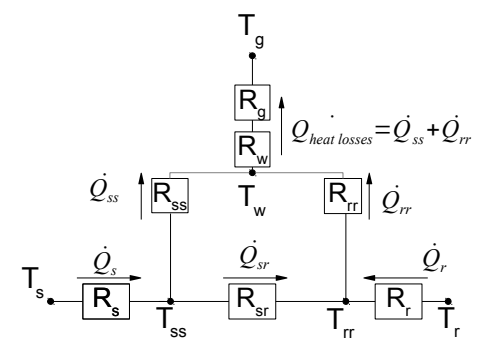

(b) Electrical equivalent

Figure 5: Trench used in the district heating network at ULg

where the suffix $h p t$ and $l p t$ refer to the high and low pressure turbine respectively, $P_{a u x}$ is the power consumed by the auxiliaries (pumps, fan,...) and $\zeta$ is the fraction of steam extracted at the back-pressure turbine outlet.

\subsection{District Heating Network}

As indicated in Figure 4, the heat produced by the CHP plant is not fully delivered to the consumption point due to the heat transfer losses across the DH network piping. The estimation of these losses is important as they can represent a significant amount of the heat produced when the thermal consumption is low (during summer time).

To introduce the discussion, a cross-section of the trench containing the supply and return pipes of the DH network is supplied in Figure 5a. District heating pipes can be insulated pipes buried in the ground or alternatively placed in a buried concrete duct. The present contribution is not intended to be a review of the literature on the calculation of heat losses in district heating and the interested reader is referred to $[15,16]$ for a more complete information.

Strictly speaking, the problem is a multi-dimensional unsteady heat transfer problem whose resolution falls beyond the scope of the present study and the problem will be treated herein as a steady-state two-dimensional conduction-convection problem. When only conduction is present, the problem of two-dimensional heat transfer between two isothermal surfaces $A_{1}$ and $A_{2}$ is generally expressed as:

$$
\dot{Q}=-\lambda \iint_{A_{1}}\left(\frac{\partial T}{\partial n}\right)_{1} d A_{1}=\lambda \iint_{A_{2}}\left(\frac{\partial T}{\partial n}\right)_{2} d A_{2}
$$

which can be simplified by the definition of a shape factor $S_{l}$ per unit length as:

$$
\dot{Q}=\lambda \cdot S_{l} \cdot l \cdot\left(T_{1}-T_{2}\right) \text { with } S_{l}=\frac{\iint_{A_{1}}\left(\frac{\partial T}{\partial n}\right)_{1} d A_{1}}{l\left(T_{2}-T_{1}\right)}
$$

The attractive property of the above formulation is that shape factors for various geometries can be found in the literature (see for example [12]). Therefore, it is possible to decompose the DH cross-section as a superposition of several elementary conduction problems each of which being modelled by a heat transfer equation of the form: 


$$
\dot{Q}=\lambda S_{l} l\left(T_{2}-T_{1}\right)=\frac{T_{2}-T_{1}}{R}
$$

In the above equation, the equivalent heat transfer resistance $R$ is defined which enables the heat transfer problem to be translated into an electrical equivalent. For example, the situation depicted in Figure 5a is represented below in Figure 5b where the DH network losses are represented as $\dot{Q}_{D H l o s s}$.

In Figure $5 \mathrm{~b}$, the resistances $R_{s}, R_{r}$ and $R_{w}$ are calculated for two concentric cylinders and adding the thermal resistance from the water to the inner tube surface when applicable. The resistance $R_{g}$ is obtained for a cylinder buried into the ground (similar formulation is available for square ducts) and the resistance $R_{s r}$ is obtained for two tubes in a medium of wide extent. If the inner space of the duct around the pipes is filled with a solid insulation material, the resistance are simply modelled by considering eccentric tubes but if this space is filled with air then convection must be taken into account. The latter problem is more difficult but an estimate of the thermal resistance can be found by using the assuming free convection around horizontal cylinders. The convective heat transfer coefficient is corrected to take into account the radiative component of the heat transfer (see [12] for details). The air temperature inside the duct is approximated as $T_{a}=\left(2 T_{d}+T_{r r}+T_{s s}\right) / 4$.

Using the Kirchhoff law, the model represented in Figure $5 \mathrm{~b}$ is solved for the temperatures $T_{r r}, T_{s s}, T_{w}$ for given value of $T_{s}, T_{r}$ and $T_{g}$ (taken equal to the ambient air temperature). The obtained model has been validated on experimental data available in the literature $([17,18]$ as well as some measurements performed on the DH network available in Liège). The interesting conclusion of this validation process is that even though the wide range of considered configurations the heat loss prediction obtained by the presented model remains within 5\% accuracy for heat losses in the range 50 to $140 \mathrm{~W} / \mathrm{m}$. The tested configurations are two pipes buried in a common cylindrical duct, two pipes in a concrete rectangular trench, two pipes in an insulated rectangular box for laboratory experiments, two buried pipes in separate ducts and the the configuration represented in Figure 5a.

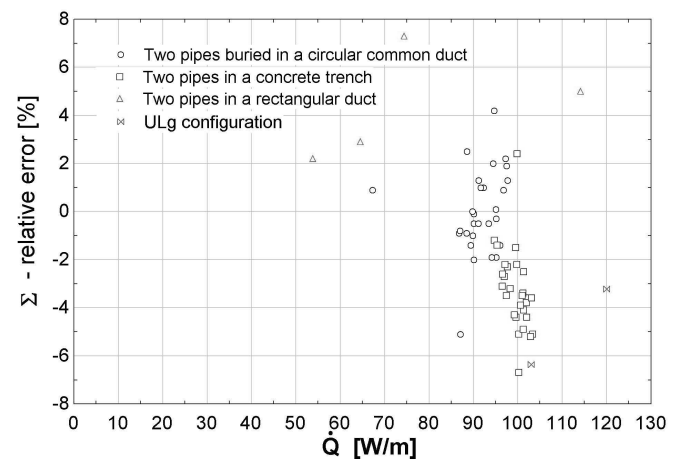

Figure 6: Prediction error between the presented model for DHN losses calculation and measured losses for several configurations. 


\section{Application}

\subsection{Description}

The aforementioned simulation model composed by the different sub-models detailed in the previous section is applied to a typical district heating application available on the University campus in Liège. The installed network has a total length of $10 \mathrm{~km}$ and distributes pressurized hot water at $125^{\circ} \mathrm{C}$ to approximately 70 buildings located in the University campus representing a total heated area of about $470000 \mathrm{~m}^{2}$. Buildings are very different in nature namely, classrooms, administratives offices, research centers, laboratories and a hospital. The hospital represents about $25 \%$ of the total heated area and requires steam for the kitchen and air humidity control system. The effective peak power of the network is around 56MW for a total of $60000 \mathrm{MWh}$ per year.

While all the buildings are heated between 4:00 to 20:00, the hospital needs heating and steam 24 hours a day, 365 days per year. The resulting daily repartition of the heat demand is similar to the one provided in Figure 2 and exhibits a peak demand at 7:00 to heat the buildings in the morning and a secondary peak around 16:00.

The DH network is operating since the 60's where the heat was generated by natural gas boilers. In order to cope with the quotas on the $\mathrm{CO}_{2}$ emissions level, the University of Liège decided to invest in biomass CHP plant whose purpose is to feed the base heat demand of the campus. The CHP plant has started full operation in 2012. It is made of a moving grid biomass boiler with nominal primary power of $12 \mathrm{MW}$ providing the steam to a back-pressure turbine and a extraction condensing turbine with nominal power of $2.4 \mathrm{MW}$. The extracted steam is condensed in a heat exchanger feeding the DH network with a nominal power of $7 \mathrm{MW}$. The remaining thermal power needed by the DH network is provided by two natural gas boilers. The primary energy is made of wood pellets whose wet basis mass composition is $46.38 \%$ of $\mathrm{C}, \mathbf{5 . 6 4 \%}$ of $\mathrm{H}, \mathbf{4 0 . 0 1}$ $\%$ of $\mathrm{O}, \mathbf{0 . 0 8 \%}$ of $\mathrm{N}, \mathbf{0 . 2 8 \%}$ of ashes, $7.6 \%$ of water and less then $0.01 \%$ of $\mathrm{S}$. Combustion model was validated with data measurements of this boiler with good agreement (Table 1).

Table 1: Comparison between experimental data and model of combustion gases composition.

\begin{tabular}{l|rr} 
& Experimental & Combustion model \\
\hline $\mathrm{CO}_{2}$ dry [\%] & $7.7 \pm 0.08$ & 8 \\
$\mathrm{O}_{2}$ dry [\%] & $12.8 \pm 0.08$ & 12.7 \\
$\mathrm{~N}_{2}$ dry [\%] & $79.7 \pm 0.08$ & 79.3 \\
$\mathrm{H}_{2} \mathrm{O}$ dry [\%] & 8.1 & 7.9 \\
$\mathrm{SO}_{2}[\mathrm{mg} / \mathrm{Nm} 3]$ & $34 \pm 11$ & 29
\end{tabular}

A schematic of the plant configuration is supplied in Figure 7.

The biomass feeding system is a moving grid in a simple combustion chamber without staged-air combustion supply. As the biomass fuel consists of wood pellets, flame temperature is too high and exhaust fumes must be recirculated and introduced after the secondary air injection. High excess air is also required to keep grid temperature in acceptable ranges. The flue gas at the exhaust of the furnace passes successively through an evaporator (platen), screen tubes, two super-heaters, one evaporator and four economizers. Exhaust gases are filtered before being directed to the stack. The steam cycle is representative of a traditional cycle with extraction turbines.

The district heating network is divided into twenty-three sections having the same geometric characteristic but pipe diameters ranging from 50 to $350 \mathrm{~mm}$. The insulation used is mineral wool with an identified thermal conductivity of $0.047 \mathrm{~W} / \mathrm{m} / \mathrm{K}$. 


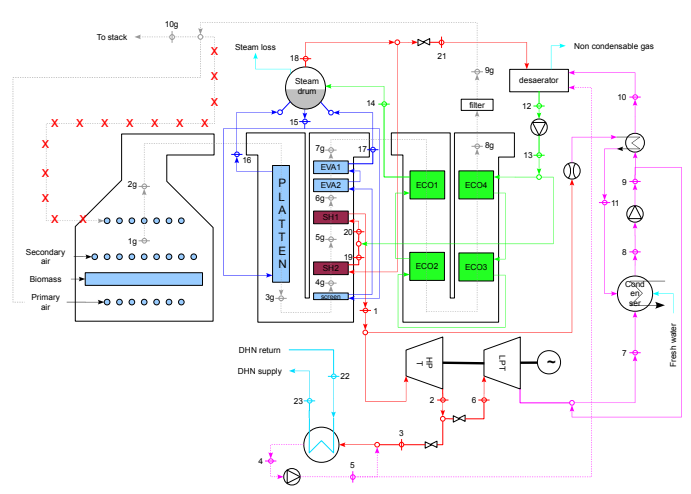

Figure 7: Schematic of CHP plant

\subsection{Model calibration}

The model detailed in section 3 is generic and must be calibrated on the existing plant in order to generate meaningful results. The simulation has been developed so as to minimize the number of parameters to be determined. In this case, the parameters to be assessed are (a) the coefficients $A U$ from relation (13) and pressure losses for the two evaporators, the screen tubes, the two super-heaters and the four economizers (b) the coefficients $K$ from relation (14) together with the turbines isentropic efficiencies and alternator efficiency, (c) pumps and fans efficiency, (d) the combustion efficiency, boiler and furnace thermal losses and (e) different bypass and recirculation steam flow rates. All these parameters have been deduced from the manufacturers data sheets enabling the model to be calibrated. Generally speaking, the model prediction is within the measurements accuracies for the steam mass flow rate and temperature, the exhaust flue gas mass flow rate and temperature and for the DH network thermal power. The model is also in good agreement with the measured flue gas composition.

The electricity production was well predicted yet with a systematic overestimation of about $4 \%$ on the whole operating range. A correction multiplying factor of $96 \%$ has been set to correct the model prediction (see Figure 8 for details).

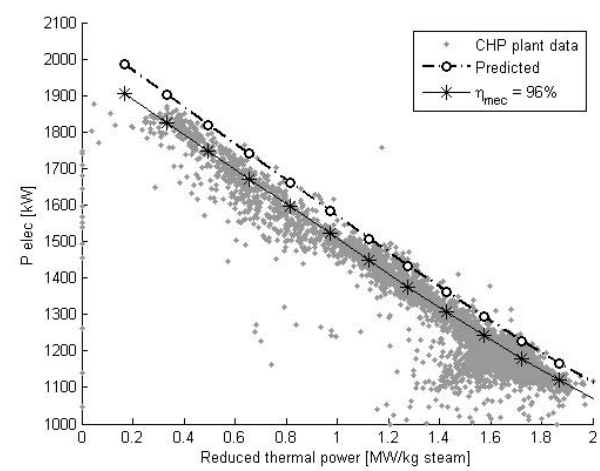

Figure 8: Calibration of the electricity production to the available measurements. 


\subsection{Simulation results}

In order to deduce results that can be extrapolated for future years, consumption data from 2009, 2010 and 2011 have been used and corrected based on the ambient temperature of the last 12 years (the degree-days method is used). Based on this study, the average thermal consumption is $60834 \mathrm{MWh}$ per year. The corresponding demand curve is similar to the one depicted in Figure 3 .

When the thermal consumption profile is fed into the simulation model of the CHP plant and DH network, the resulting CHP thermal production is $36394 \mathrm{MWh}$ while $8945 \mathrm{MWh}$ of electricity are generated. This represents approximately $60 \%$ of the thermal needs of the University campus. On average, the thermal and electrical efficiencies are respectively $38.1 \%$ and $9.4 \%$ giving a total efficiency $\eta_{c h p}=47.5 \%$. Despite the fact that the studied CHP plant has a nominal efficiency of about $75 \%$, similar to other conventional cogeneration plants, this efficiency decreases when the mass flow rate of steam extracted from the turbine decreases (i. e. heat production decreases). In the limit, when no heat is produced, the global efficiency reaches the maximal electrical efficiency (around 20\%). Therefore the efficiency mentioned in this section are average efficiencies embedding part load operation. Based on the thermal energy and installed power, the equivalent utilization time can be assessed which gives $\tau_{e}=5200 \mathrm{~h} / \mathrm{y}$. In the above results it has been assumed that the plant is stopped 21 days during summer time and a reliability factor of $2 \%$ for the remaining time of the year.

An interesting outcome of the model is the assessment of the total heat losses from the DH network which are estimated at 5212 MWh per year thus representing $8.5 \%$ of the total heat generated by both the CHP plant and backup boilers. The heat losses averaged on a daily basis are represented in Figure 9 which exhibits the different loss patterns for summer time (lower cloud) when only the hospital is supplied and winter time (upper cloud) when the whole campus is heated.

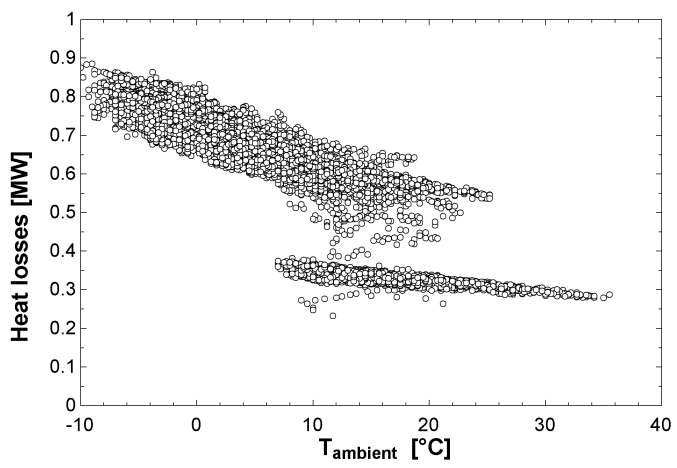

Figure 9: Simulated DHN total heat losses versus the ambient temperature using the model developed in section 3.4.

From the environmental point of view, the CHP plant allows a significant saving in terms of $\mathrm{CO}_{2}$ emissions. indeed, when compared to the separate generation of electricity and heat, where electricity is supposed generated by natural gas combined cycle power plant with average efficiency of $55 \%$ thus emitting $456 \mathrm{~kg}$ of $\mathrm{CO}_{2}$ per MWh and thermal energy produced by a natural gas boiler with $90 \%$ efficiency thus emitting $279 \mathrm{~kg}$ of $\mathrm{CO}_{2}$ per MWh, the CHP plant reduces the $\mathrm{CO}_{2}$ emissions of about 12800 tons per year. With respect to the total energy consumption of the University campus, this represents a reduction of $38 \%$ of the $\mathrm{CO}_{2}$ emissions. 


\subsection{Cost of Heat}

Besides the environmental and energetic study it is worth looking at the economic side of the problem. To that end, 3 basic configurations are considered, namely:

1. the heat is produced in every single building by natural gas hot water boilers,

2. the heat is produced by a natural gas hot water boiler and delivered by the district heating network,

3. the heat is produced by the CHP plant together with the natural gas backup boilers and delivered by the district heating network.

These configurations are limited as the focus of this article is on $\mathrm{CO}_{2}$ emissions reduction for previous and current situation of ULg heat production.

Based on the simulation results already presented, it is possible to use relations (2) and (6) to assess the unit cost of heat provided that other data such as investment, operation and maintenance costs plus the primary energy cost are available. Reliable data can be easily found in the literature, for example the interested reader is referred to $[9,19]$. The data used herein are summarized in Table 2.

\begin{tabular}{l|ccc} 
& $C$ & $U_{\text {fix }}$ & $u_{\text {var }}$ \\
\hline & $(€ / \mathrm{kW})$ & $(€ / \mathrm{y} / \mathrm{kW})$ & $(€ / \mathrm{MWh})$ \\
CHP & 3000 & 150 & 5 \\
NG boiler $(<1 \mathrm{MW})$ & 25 & 6 & 5 \\
NG boiler $(>1 \mathrm{MW})$ & 20 & 4 & 3
\end{tabular}

Table 2: Cost data for the calculation of the $\mathrm{COH}$ (for the CHP plant the costs are mentioned per electrical installed power rather than thermal power.

The lifetime of the installation is taken to be equal to 20 years with a discount rate of $5 \%$. Concerning the DH network, the investment cost is considered to be $250 € / \mathrm{m}$ with a lifetime of 50 years. The fuel cost is $33 € /$ MWh for the biomass (wood pellets) and $36.55 € / \mathrm{MWh}$ for the natural gas. For the test case 1 where the heat is produced locally, the natural gas costs $55.9 € / \mathrm{MWh}[3]^{4}$. The electricity produced by the CHP plant is used by the University and accounted at the value of $112.7 € /$ MWhe. Based on the actual $\mathrm{CO}_{2}$ savings, approximately 3 green certificates should be allocated per every MWh of electricity produced by the CHP plant, however the maximum number of green certificates allowed per MWh of electricity is two. Green certificates are supposed to have a value of $75 €$.

The resulting breakdown of the cost for the different test cases are summarized in Table 3 . The table gathers unit cost of heat split up into fixed, variable and electricity selling contribution. The part coming from the investment of the DH network is also mentioned. For test case 3, the costs related to the backup boilers and CHP and mentioned and the average is assessed through relation (4).

The first conclusion to be drawn is that the heat from the district heating network is cheaper than from a natural gas boiler and this even if DH network transport losses are taken into account. The most important difference is of course the selling of the electricity which decrease the cost of heat generated by the CHP plant. On the other hand, when comparing the backup natural gas boilers, the table exhibits that the heat generated by the CHP plant is not economically viable even if the quotas on the emissions level are considered ${ }^{5}$.

\footnotetext{
${ }^{4}$ The energy cost data are extracted from the Eurostat web site for the Belgian market in 2011.

${ }^{5} \mathrm{~A}$ selling price of $17.9 € / \mathrm{t} \mathrm{CO}_{2}$ is necessary to make CHP plant economically viable whereas it is currently $6 € / \mathrm{t} C \mathrm{O}_{2}$. Notice the price of the carbon ton was quite high (about $€ / \mathrm{t} C \mathrm{O}_{2}$ ) when the plant was designed. Morevover the biomass
} 


\begin{tabular}{l||r|r|rrr} 
(in $€ / M W h)$ & case 1 & case 2 & \multicolumn{3}{|c}{ case 3 } \\
& & & NG boilers & CHP & average \\
\hline \hline Fixed & 7.52 & 5.76 & 12.81 & 28.18 & \\
Variable & 70.76 & 50.31 & 50.31 & 100.10 & \\
Electricity & 0.00 & 0.00 & 0.00 & -70.63 & \\
DHN & 0.00 & 2.25 & 2.25 & 2.25 & \\
\hline Total & $\mathbf{7 8 . 2 8}$ & 58.32 & 65.37 & 59.90 & $\mathbf{6 2 . 0 9}$
\end{tabular}

Table 3: Breakdown of the cost of heat

\section{Improvement scenarios}

The study of the cost of heat is interesting to simply monitor the profitability of a given investment, however, the most important aspect of the methodology is its ability to investigate saving measures.

\subsection{Excess air decrease}

One of the most evident way to decrease the cost of heat is to improve the conversion efficiency of the biomass into heat and electricity. As already mentioned before, the excess air of the existing is important to guarantee sufficient cooling of the moving grid in the furnace which generates low boiler efficiency. Decreasing the excess air together with a recirculation of the flue gas at the level of the primary air rather than after the secondary air enables a better boiler efficiency and a more efficient use of the biomass. Besides, the higher efficiency, it can be expected than $N O_{x}$ emissions will decrease when using flue gas recirculation with the combustion air.

Using the aforementioned model of the plant makes it relatively easy to simulate the resulting boiler efficiency on the whole operating range of the plant and thus studying the influence on the cost of heat. Practically, the flue gas recirculation enables an improvement of the boiler efficiency of around 4\% which in turn improves the electrical and thermal efficiency respectively to $9.6 \%$ and $39 \%$. The resulting decrease in the cost of heat is around $1.35 € / \mathrm{MWh}$ to reach $60.74 € / \mathrm{MWh}$

\subsection{Regenerative heat exchanger}

While the previous improvement scenario enhances the boiler efficiency by decreasing the flue gas rejected to the atmosphere, another way to improve the plant consists in installing a regeneration heat exchanger intended to preheat the combustion air by cooling the flue gas at the exhaust. The actual exhaust temperature being around $170^{\circ} \mathrm{C}$, a temperature difference of $20^{\circ} \mathrm{C}$ is available until the acid dew point is reached $\left(150^{\circ} \mathrm{C}\right.$ for biomass boilers). If the regenerator efficiency is supposed equal to $87 \%$ (see [20]) a power of $150 \mathrm{~kW}$ can be transferred to the combustion air thus increasing the boiler efficiency by another $1.3 \%$. The boiler efficiency increase is somehow balanced with an increase fan consumption due to the increased pressure losses in the combustion air supply line and flue gas recirculation. Due to the latter remark, the additional decrease in the cost of heat is moderate and around $0.52 € / \mathrm{MWh}$ if the two improvements are combined. The cost of heat evolution of the two previous improvements are summarized in Figure 10.

fuel price has increased more as expected whereas the natural gas price decreased. For all these reasons, the cost of heat of the CHP plant is currently higher than natural gas boilers solution. 


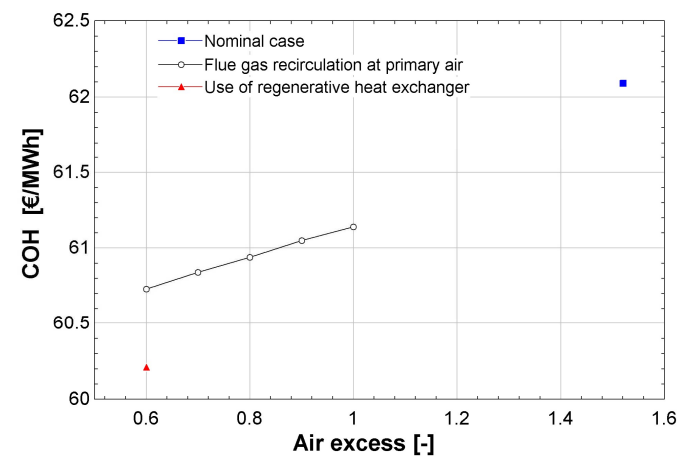

Figure 10: Cost of heat for several conditions as a function of excess air.

\subsection{Reduction of heat transport losses}

The third scenario considers the decrease of the transport heat losses by decreasing the temperature of the circulating water. To support the discussion, it is convenient to consider a simple model for the average heat losses per unit length of DH pipes:

$$
\dot{q}_{D H l o s s}=\Lambda \cdot\left(T_{f d}-T_{a m b}\right)
$$

where $T_{f d}$ is an average temperature of the water circulating in the DH network, $T_{a m b}$ is the ambient temperature and $\Lambda$ is a global heat transfer coefficient per unit length. To identify the global heat transfer coefficient, the daily heat losses from Figure 9 are plotted against the temperature difference $T_{f d}-T_{a m b}$ and corrected to take into account the effective exchange surface coming from the different operation of the district heating network (all the branches are not used during the whole year). The resulting trend is represented in Figure 11 where it can be seen that the two feeders have similar transfer coefficients (the slope of the resulting line is similar). The slight difference can be explained by the different level of temperature in the two feeders which can be responsible of a drift in the pipe insulation thermal conductivity (see [21] for more details).

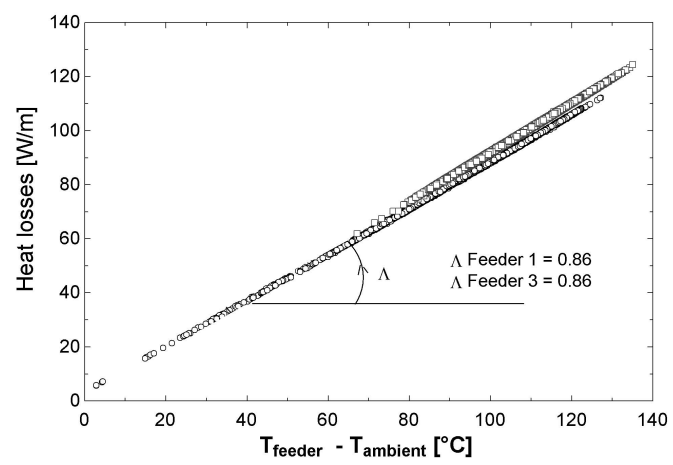

Figure 11: Simulated heat losses corrected by the effective heating area as a function of the temperature difference between feeder and ambient temperature. 
From the above discussion, it is clear that decreasing the temperature of the water circulating into the network by $40^{\circ} \mathrm{C}$ saves half of the energy lost in the heat transport. A detailed calculation by the simulation gives a saving of $2010 \mathrm{MWh}$ per year. For sake of comparison, changing the pipe insulation from mineral wool to PUR would save only $1450 \mathrm{MWh}$ per year. The effect of decreasing the water temperature by $40{ }^{\circ} \mathrm{C}$ generates an additional saving in the cost of heat of about $0.81 € / \mathrm{MWh}$ compared to the decrease of air excess.

\section{CONCLUSIONS}

Analysing the opportunity to invest in a CHP plant or monitoring the efficiency of a CHP plant connected to a DH network is not an easy task as energetic, environmental and economic aspects must be combined for the whole operation envelope of the plant. This contribution presents a synthetic way to achieve such a task using only simple models from thermodynamic, combustion process, heat transfer and finance. The interesting aspect is that a somehow generic combustion model is coupled to a boiler and district heating network covering the whole supply chain from the production to the transport of the energy and taking into account the specific profile of the heat demand.

The resulting cost and environmental information constitutes a reliable and meaningful way of presenting the main characteristics of a specific application which may dramatically improves the decision making process. The basic information required is not very important and may, most of the time, be found in the existing literature. The only specific information used herein is the heat consumption data. These data must be carefully analyzed as the influence of the CHP plant part load operation is very important. It is important to note that, depending on the annual heat demand characteristic, the annual average efficiency can be much lower than the nominal efficiency thus leading to an increased cost of heat. The conclusions in terms of cost are representative of the presented application but can be easily extended to other countries by substituting energy costs and subsidizing policies.

In order to improve the estimation of the environmental impact, incomplete combustion models should be integrated so as to forecast pollutants emissions $\left(C O, N O_{x}, \ldots\right)$. Also heat storage is not considered herein which could dramatically improve the profitability of the CHP plant by increasing its equivalent utilization time. Finally, the dynamic behaviour of the DH network is neglected in the present study. Taking into account dynamic effect could improve the efficiency assessment yet with an important increase of the model complexity.

[1] Varun, I. Bhat, R. Prakash, Lca of renewable energy for electricity generation systems—a review, Renewable and Sustainable Energy Reviews 13 (2009) 1067 - 1073.

[2] H. Lund, B. Moller, B. Mathiesen, A. Dyrelund, The role of district heating in future renewable energy systems, Energy 35 (2010) 1381 - 1390.

[3] EUROSTAT, http://ec . europa. eu/eurostat, 2013. Last consulted the 26 January 2013.

[4] N. T. Raj, S. Iniyan, R. Goic, A review of renewable energy based cogeneration technologies, Renewable and Sustainable Energy Reviews 15 (2011) 3640 - 3648.

[5] T. Savola, I. Keppo, Off-design simulation and mathematical modeling of small-scale chp plants at part loads, Applied Thermal Engineering 25 (2005) 1219 - 1232.

[6] L. Barelli, G. Bidini, E. Pinchi, Implementation of a cogenerative district heating: Optimization of a simulation model for the thermal power demand, Energy and Buildings 38 (2006) $1434-1442$.

[7] M. Pirouti, J. Wu, J. Ekanayake, N. Jenkins, Dynamic modelling and control of a direct-combustion biomass chp unit, in: Universities Power Engineering Conference (UPEC), 2010 45th International, 2010, pp. 1 -6.

[8] R. Bachmann, H. Nielsen, J. Warner, Combined - Cycle Gas \& Steam Turbine Power Plants, Pennwell Books, Tulsa, Oklahoma, 1999. URL: .

[9] E. Styrelsen, Technology data for energy plants: Generation of Electricity and District Heating, Energy Storage and Energy Carrier Generation and Conversion, Technical Report, Energi Styrelsen, 2012. 
[10] S. v. Loo, J. Koppejan, The Handbook of Biomass Combustion and Co-firing, reprint ed., Routledge, 2010.

[11] M. Moran, H. Shapiro, Fundamentals of engineering thermodynamics, 6 ed., John Wiley and Sons Inc., New York, NY, 2009. URL: http://eu.wiley.com/WileyCDA/WileyTitle/productCd-0470540192.html.

[12] V.-G. V. und Chemieingenieurwesen (Ed.), VDI heat atlas, Springer, 2010. 2nd edition.

[13] R. Shah, D. Sekulić, Fundamentals of heat exchanger design, Wiley, 2003.

[14] L. C. L. A. Stodola, Steam and gaz turbines, volume 1, McGraw-Hill, 1927.

[15] B. Bøhm, On transient heat losses from buried district heating pipes, International Journal of Energy Research 24 (2000) 1311-1334.

[16] W. P., Steady-state heat loss from insulated pipes, Ph.D. thesis, Department of Building Physics, Lund Institute of Technology, Sweden, 1991

[17] G. E. Phetteplace, M. J. Kryska, D. L. Carbee, Field Measurements of Heat Losses from Three Types of Heat Distribution Systems, Technical Report, COLD REGIONS RESEARCH AND ENGINEERING LAB HANOVER NH, 1991.

[18] P. W. Richmond, Two-dimensional analysis of natural convection and radiation in utilidors, US Army Corps of Engineers (1999).

[19] G. Streckiene, A. N. Andersen, Analyzing the optimal size of CHP-Unit and thermal store when a German CHPplant is selling at the Spot market, Technical Report v1.2, EMD International A/S, 2008

[20] C. F. McDonald, Recuperator considerations for future higher efficiency microturbines, Applied Thermal Engineering 23 (2003) 1463 - 1487.

[21] H. M.-S. F. Ochs, Temperature and moisture dependence of the thermal conductivity of insulation materials, NATO Advanced Study Institute on Thermal Energy Storage for Sustainable Energy Consumption (TESSEC) (2005). 